\title{
Cardiomegalia associada ao hipotireoidismo clínico
}

\author{
Cardiomegaly associated with clinical hypothyroidism
}

\author{
Tiago Cardoso Martinelli ${ }^{1}$, Renan Corrêa Braga ${ }^{1}$, Bárbara Ahnert Blanco de Moura \\ Magalhães ${ }^{1}$, Sofia Villela Musso', Regina Célia Tonini², Rachel Torres Sasso ${ }^{3}$, \\ Carmen Dolores Gonçalves Brandão ${ }^{4}$
} Martinelli TC, Braga RC, Magalhães BABM, Musso SV, Tonini RC, Sasso RT, Brandão CDG. Cardiomegalia associada ao hipotireoidismo
clínico / Cardiomegaly associated with clinical hypothyroidism. Rev Med (São Paulo). 2019 jan.-fev.;98(1):81-5.

RESUMO: Introdução: O hipotireoidismo primário corresponde a 95\% do total de casos de hipotireoidismo. Caracteriza-se como uma síndrome clínica onde o hormônio estimulante da tireoide (TSH) encontra-se acima dos valores de referência e a tiroxina livre (T4 L) abaixo do limite da normalidade. Essas alterações levam a uma diminuição da taxa metabólica basal o que pode acometer o aparelho cardiovascular. Casos severos podem levar a um derrame pericárdico e cardiomegalia. Este relato de caso descreve uma manifestação atípica do hipotireoidismo, o derrame pericárdio, como um evento inicial da doença, e visa contribuir para a literatura médica, discutindo a propedêutica realizada para diagnóstico e descrevendo a resposta terapêutica e evolução clínica. Relato de Caso: sexo feminino, 44 anos, parda, história de hipotireoidismo em tratamento irregular veio encaminhada para um exame eletivo de tomografia computadorizada do abdome em investigação de nefrolitíase, porém neste evidenciou-se um importante derrame pericárdico sem comprometimento hemodinâmico. À radiografia de tórax evidenciou aumento da área cardíaca e o ecocardiograma evidenciou uma efusão pericárdica (aspecto swinging heart), movimento paradoxal de septo interventricular. O eletrocardiograma não demonstrou alterações. Durante a investigação evidenciou T4 L inferior a 0,4 ng/dL, TSH: $319,9 \mathrm{uUI} / \mathrm{mL}$, anticorpos anti-tireoperoxidase (anti-TPO):142,3UI/ ml. Avaliação reumatológica descartou doenças. Diante da hipótese de derrame pericárdico devido ao hipotireoidismo realizou então o tratamento com levotiroxina chegando à dose de $125 \mathrm{mcg} / \mathrm{dia}$. Durante os 7 meses do tratamento houve uma regressão da cardiomegalia e do derrame pericárdico. Os exames de janeiro/2018 corroboram a hipótese evidenciando níveis de T4 L: 1,2; TSH: 2,2 com os exames de imagens normais. Conclusão: Diante da raridade do caso, este trabalho mostra uma paciente oligossintomática, mas com grande repercussão em relação à manifestação cardiológica. Com isso, os autores ressaltam a importância da investigação da função tireoidiana em pacientes com alteração cardíaca.

Descritores: Hipotireoidismo; Derrame pericárdico; Cardiomegalia.

\begin{abstract}
Introduction: Primary hypothyroidism corresponds to $95 \%$ of the total cases of hypothyroidism. Primary hypothyroidism is characterized as a clinical syndrome in which thyroid stimulating hormone (TSH) is above the reference values and free thyroxine (T4 L) below the limit of normality. These changes lead to a decrease in the basal metabolic rate that may affect the cardiovascular system. Severe cases can lead to a pericardial effusion and cardiomegaly. This case report describes an atypical manifestation hypothyroidism, the pericardial effusion, as an initial event of the disease, and aims to contribute to the medical literature, discussing a propaedeutic performed for diagnosis and describing a therapeutic response and clinical evolution. Case Report: A 44-year-old female patient; history of hypothyroidism undergoing irregular treatment. She was referred for elective computed tomography examination of the abdomen in nephrolithiasis investigation, but an important pericardial effusion was demonstrated without hemodynamic compromise. The chest $\mathrm{X}$-ray showed an increase in the cardiac area and the echocardiogram showed a pericardial effusion (swinging heart aspect), paradoxical movement of the interventricular septum. The electrocardiogram showed no changes. During the investigation, T4 L was lower than $0.4 \mathrm{ng} / \mathrm{dL}$, TSH: $319.9 \mathrm{uUI} / \mathrm{mL}$, anti-hyroperoxidase: $142.3 \mathrm{IU} / \mathrm{ml}$. Rheumatologic evaluation ruled out diseases. Faced with the hypothesis of pericardial effusion due to hypothyroidism, the patient then underwent levothyroxine treatment at a dose of $125 \mathrm{mcg} /$ day. Through 7 months of treatment there was a regression of cardiomegaly and pericardial effusion. The exams of January/2018 corroborate the hypothesis evidencing levels of T4 L: 1,2; TSH: 2.2 with normal imaging tests. Conclusion: In view of the rarity of the case, this work shows an oligosymptomatic patient, but with great repercussion in relation to the cardiological manifestation. The authors emphasize the importance of investigating thyroid function in patients with cardiac alterations.
\end{abstract}

Keywords: Hypothyroidism; Pericardial effusion; Cardiomegaly.

Apresentado como Pôster - Clínico, XVIII Encontro Brasileiro de Tireoide, Campos do Jordão, SP, 19-22 abr. 2018.

1. Acadêmicos de Medicina da Escola Superior de Ciências da Santa Casa de Misericórdia de Vitória, EMESCAM, Vitória, ES. ORCID: Martinelli TC - https://orcid.org/0000-0001-6397-8805; Braga RC - https://orcid.org/0000-0001-5457-6089; Magalhães BABM - https://orcid.org/0000-0001-56919145; Musso SV - https://orcid.org/0000-0002-5587-9644.Email: tiago_cm_@hotmail.com; barbara.abmm96@gmail.com; sofiavmusso@gmail.com.

2. Especialista em Clínica Médica, Professora Assistente da Escola Superior de Ciências da Saúde da Santa Casa de Misericórdia de Vitória, EMESCAM, Vitória, ES. ORCID: https://orcid.org/0000-0002-8826-2309. Email: reginatonini@hotmail.com.

3. Endocrinologista, Professora Assistente da Escola Superior de Ciências da Saúde da Santa Casa de Misericórdia de Vitória, EMESCAM, Vitória, ES. ORCID: https://orcid.org/0000-0002-6039-5572. Email: rachelts@ibest.com.br.

4. Endocrinologista, Orientadora deste trabalho, Professora Adjunta da cadeira de Endocrinologia e Metabologia da Escola Superior de Ciências da Saúde da Santa Casa de Misericórdia de Vitória, EMESCAM, Vitória, ES. ORCID; https://orcid.org/0000-0003-3239-9979. Email: loloi@terra.com.br. Endereço para correspondência: Tiago Cardoso Martinelli. Rua Celso Calmon, nº 445, apto 1002. Ed. De Biase, Bairro Praia do Canto. Vitória, ES. CEP: 29055-590. Email: tiago_cm_@hotmail.com. 


\section{INTRODUÇÃO}

hipotireoidismo caracteriza-se como uma
síndrome clínica que culmina no alentecimento generalizado dos processos metabólicos, devido à diminuição ou ação dos hormônios tireoidianos ${ }^{1}$. Pode ser classificado de acordo com qual órgão do eixo hipotálamohipófise-tireóide foi acometido (terciário, secundário ou primário, respectivamente) ${ }^{1}$.

Devido à grande variação na apresentação clínica e ausência de sintomas específicos, a definição dessa doença é predominantemente bioquímica. O hipotireoidismo clínico primário é definido pela presença do hormônio estimulante da tireóide (TSH) acima dos valores de referência e tiroxina livre (T4 L) abaixo do limite da normalidade ${ }^{2}$. Essa patologia acomete, sobretudo, mulheres e indivíduos com idade superior a 65 anos, sendo ainda mais comum associado a outras doenças autoimunes ${ }^{2}$.

O hipotireoidismo primário corresponde a $95 \%$ dos casos. Em países no qual não há deficiência de iodo a Tireoidite de Hashimoto é a principal causa de comprometimento tireoidiano e é caracterizada por lesão autoimune decorrente da estimulação e produção de células B e plasmócitos que produzem anticorpos contra antígenos tireoidianos ${ }^{1,3}$. Fator essencial para definição dessa etiologia é a presença dos anticorpos anti-tireoglobulina (anti-Tg) e/ou antitireoxidade (anti-TPO), encontrados em 95\% dos pacientes com Tireoidite de Hashimoto ${ }^{3}$.

A menor taxa de hormônios tireoidianos circulantes possui impacto negativo na taxa metabólica basal o que repercute em diversos órgãos e sistemas ${ }^{4}$. Sintomas como pele seca, fadiga, intolerância ao frio e alterações na voz destacam-se como os mais prevalentes ${ }^{1,4,5}$.

Especificamente do aparelho cardiovascular, o hipotireoidismo possui repercussões de impacto na hemodinâmica e função cardíaca, podendo estar correlacionado ao menor débito cardíaco, aumento da resistência vascular sistêmica, aumento da pressão arterial diastólica e maior predisposição à aterosclerose. $\mathrm{O}$ hipotireoidismo severo pode estender o comprometimento cardíaco levando a uma cardiomegalia (devido ao mioedema) e, também, para região pericárdica, manifestando-se sob a forma de derrame pericárdico. Trata-se de um evento raro na literatura médica cujo mecanismo pode ser explicado pelo aumento da permeabilidade capilar e redução da drenagem linfática da região do pericárdio, devido a um estágio avançado do hipotireoidismo não tratado ${ }^{6,7}$.

O comprometimento cardíaco pode ter maior gravidade. Apenas 20 casos de hipotireoidismo com tamponamento cardíaco foram relatados ${ }^{8}$. A prevalência de derrame pericárdico em pacientes com hipotireoidismo varia de $30 \%$ a $88 \%$, segundo a literatura 9 . O fato de poucos casos de tamponamento cardíaco ocorrerem em pacientes com hipotireoidismo e derrame pericárdico deve-se ao fato de que o acúmulo de líquido é lento, o que permite uma melhor complacência pericárdica ao longo do tempo sem que haja comprometimento hemodinâmico ${ }^{8,9,10}$.

O hipotireoidismo é caracterizado pela boa resposta terapêutica a reposição hormonal com Levotiroxina ${ }^{1,2,11}$. Peculiaridades quanto a manejo dessa medicação devem ser individualizadas conforme presença de outras comorbidades associadas e medicações em uso, por interferir na absorção da levotiroxina ${ }^{11}$.

\section{RELATO DO CASO}

Paciente do sexo feminino, 44 anos, parda, casada, do lar, com história prévia de hipotireoidismo em tratamento irregular - estava há 05 anos sem usar a medicação (utilizava a dose de $150 \mathrm{mcg}$ ) e retomou o uso em subdose $(50 \mathrm{mcg} / \mathrm{dia})$, por conta própria, há 2 meses e de frequência irregular.

Compareceu ao Hospital Santa Casa de Misericórdia de Vitória a fim de realizar, eletivamente, exame de tomografia computadorizada da região abdominal em junho de 2017, como parte de confirmação de doença litíasica renal. Durante a realização do exame, constatouse a presença de microlitíases não obstrutivas em rim direito e nos primeiros scouts tomográficos evidenciou-se importante derrame pericárdico (Figura 1) na paciente sem comprometimento hemodinâmico evidente.

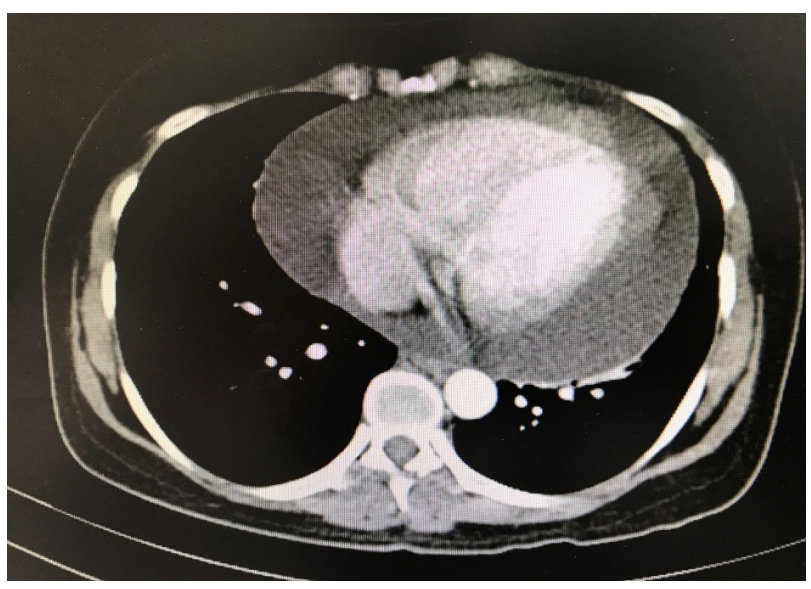

Figura 1. Derrame pericárdico visto em tomografia computadorizada

Com finalidade de avaliação etiológica e médica complementar do derrame pericárdio, a paciente foi internada no setor de clínica médica do Hospital. Queixavase apenas de leve adinamia e letargia.

Ao exame físico: BEG, lote, afebril, mucosas hidratadas e normocoradas. Edema discreto de face e região labial, fragilidade ungueal e macroglossia. Constatouse a presença de melasma em região malar e lesões hiperemiadas e descamativas isoladas, porém distribuídas em todo corpo, sobretudo em topografia de antebraços e 
membros inferiores (clinicamente sugestiva de psoríase). Não apresentava outras alterações ao exame do aparelho respiratório e cardiovascular:

ACV: Ritmo cardíaco em 2 tempos, bulhas normofonéticas e sem sopros. $\mathrm{FC}=80 \mathrm{bpm}, \mathrm{PA}=110 \mathrm{x}$ $80 \mathrm{mmHg} . \mathrm{AR}=$ atípico, murmúrio vesicular fisiológico bilateralmente, sem ruídos adventícios, eupneica em ar ambiente e FR=18 irpm. Ao exame abdominal: abdome flácido, ruídos hidroaéreos presentes, indolor à palpação superficial e profunda, sem visceromegalias. Sem edemas, em membro inferiores, panturrilhas livres, sem empastamento.

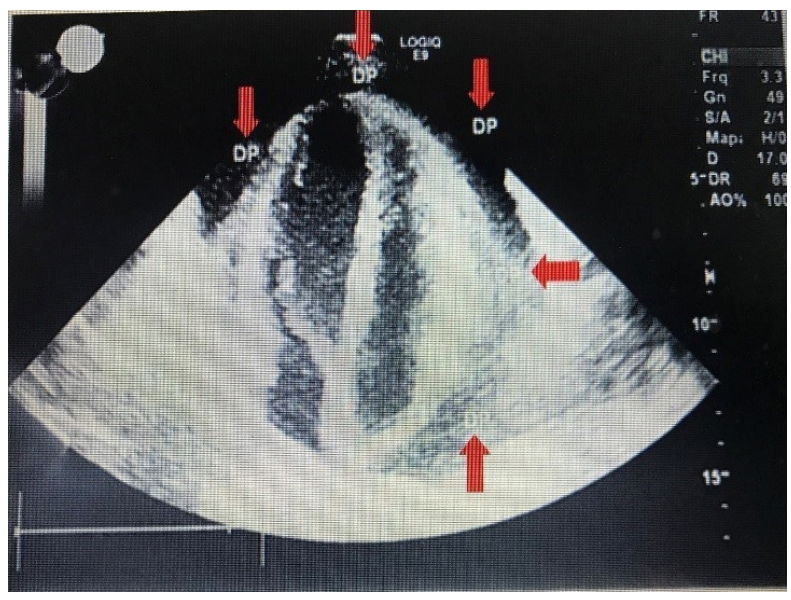

Na história patológica pregressa informa nefrolitíase e psoríase vulgar. Seu histórico familiar evidenciou mãe com hipertensão arterial e hipotireoidismo.

Complementando a avaliação cardiológica o exame de ecocardiografia corroborou a existência da efusão pericárdica (coração aspecto swinging heart) (Figura 2A), movimento paradoxal do septo interventricular e demonstrou característica anatomofuncionais normais das válvulas e câmaras cardíacas. O RX Tórax evidenciou aumento de área cardíaca (Figura 3A). O exame eletrocardiográfico da mesma forma não continha alterações isquêmicas ou distúrbios de condução, apenas baixa voltagem.

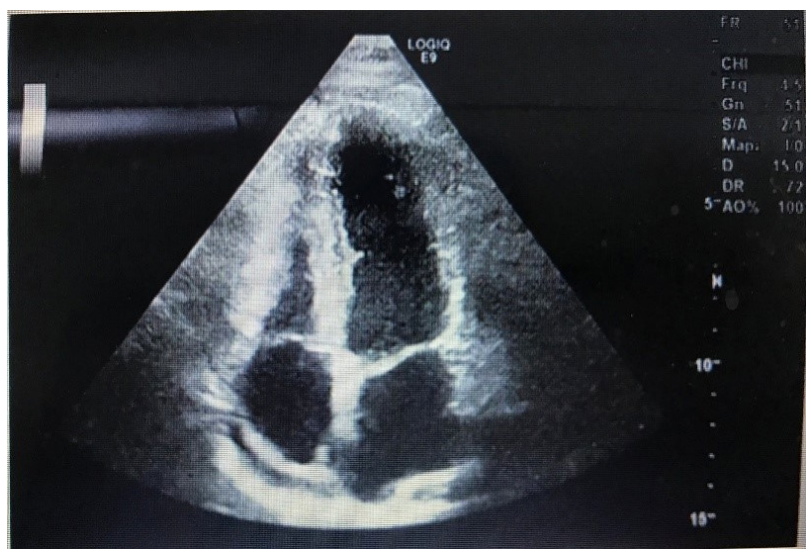

Figura 2. Ecocardiograma transtorácico. A: Derrame pericárdico. B: Resolução do derrame após o tratamento
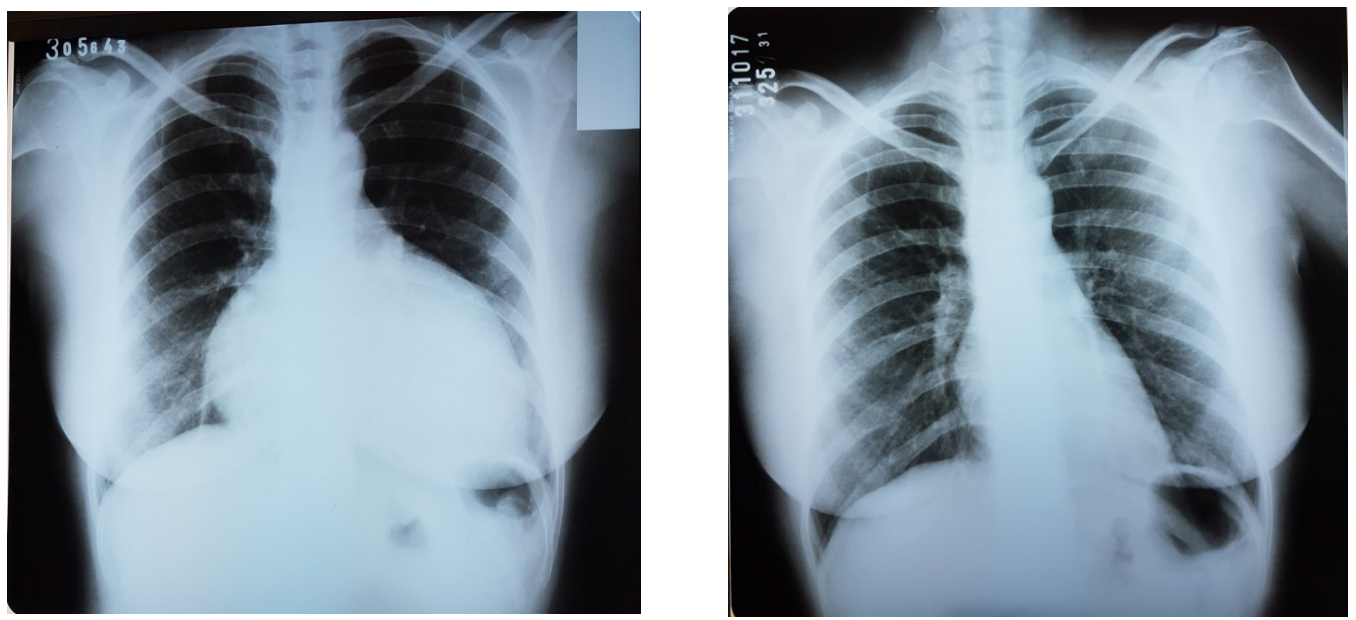

Figura 3. Radiografia de tórax. A: Aumento de área cardíaca. B: Normalização após o tratamento

Diante do quadro clínico apresentado foi iniciado avaliação a fim de indicar a etiologia desse processo. Visto perfil da paciente buscou-se laboratorialmente por causas endocrinológicas ou reumatológicas. Constataramse sorologias negativas para hepatites $\mathrm{B}$ e C; HIV; HTLV; prova inflamatória negativa (proteína $\mathrm{C}$ reativa: 7,2); Exames hematológicos sem distúrbios anêmicos, coagulação ou proliferativos; creatinina, ureia plasmática e proteinúria estavam dentro dos parâmetros normais. Das provas reumatológicas solicitadas: complemento sérico total, C3 e C4, HLA-B27, fator reumatoide, anticorpos anti-RNP, anti-LA (SSB), anti-RO (SSA), anticardiolipina $\operatorname{IgM}$ e IgG, anti-SM, anti-DNA foram negativos e/ou não atingiram valores representativos para estabelecer valor diagnóstico. O Fator anti-nuclear apresentou padrão misto nucleolar pontilhado fino com fluorescência no aparelho mitótico num título de 1:320. O screening endocrinológico destacou-se nesse contexto: observaram-se valores de T4 L inferiores a 0,4 ng/dL (VR: 0,7 - 1,48ng/dL), TSH: 319,9 uUI/mL (VR: 0,34 - 5,6), anticorpos anti- 
tireoperoxidase (anti-TPO) de 142,3 UI/ml (VR: < 9,0 $\mathrm{UI} / \mathrm{mL}$ ) vide Tabela 1 . Tais achados tornaram a principal hipótese do derrame pericárdico o hipotireoidismo, visto que a punção pericárdica foi contraindicada em função da estabilidade hemodinâmica da paciente. A paciente continuou acompanhamento ambulatorial após a alta a fim descartar causas reumatológicas e observar a possível regressão da efusão pericárdica quando reinstituída a terapia com levotiroxina gradativamente até a dose de $125 \mathrm{mcg} / \mathrm{dia}$ (esquema atual). A radiografia de tórax (Figura
2B) e ecocardiografia (Figura 3B), evidenciaram regressão do derrame e melhora dos sintomas de hipotireoidismo que a paciente apresentava (sonolência e adinamia), após sete meses de internação hospitalar e tratamento de hipotireoidismo. Exames laboratoriais de janeiro/2018 corroboram a hipótese, evidenciando níveis de T4 L:1,2, TSH: 2,2. A paciente segue em acompanhamento ambulatorial no Serviço de Endocrinologia do Hospital Santa Casa de Misericórdia de Vitória (ES).

Tabela 1. Acompanhamento das dosagens dos hormônios tireoidianos

\begin{tabular}{|c|c|c|c|c|}
\hline DIA & TSH & T4 LIVRE & ANTI-TPO & DOSE LT4 \\
\hline $16 / 06 / 17$ & $>100,0$ & - & - & $50 \mathrm{MCG}$ \\
\hline $21 / 06 / 17$ & 319,9 & 0,44 & 142 & $50 \mathrm{MCG}$ \\
\hline $10 / 08 / 17$ & 29,7 & 1,47 & - & $75 \mathrm{MCG}$ \\
\hline $27 / 09 / 17$ & 7,0 & 1,00 & - & $100 \mathrm{MCG}$ \\
\hline 09/01/18 & 2,2 & 1,2 & - & $125 \mathrm{MCG}$ \\
\hline
\end{tabular}

\section{DISCUSSÃO}

O derrame pericárdico (DP) associado ao hipotiroidismo representava um evento conhecido na literatura. No entanto, atualmente é pouco frequente devido ao fácil acesso as dosagens hormonais disponibilizadas e maior conhecimento do hipotiroidismo, fazendo diagnóstico mais frequente em fase inicial da doença. Em relação sobre a incidência pode situar entre 30 a 80 \%, mas os dados são conflitantes ${ }^{9}$.

A causa do DP deve-se ao acúmulo extravascular de albumina, devido o aumento da permeabilidade capilar no hipotiroidismo, somada a drenagem linfática insuficiente ${ }^{12}$. O lento acúmulo de líquido e a distensibilidade cardíaca podem justificar a pobreza sintomatológica da nossa paciente.

O tamponamento cardíaco é uma complicação rara, visto as características fisiopatológicas no desenvolvimento do DP, sendo típico de pacientes com hipotireoidismo sintomático de longa data ${ }^{13,14}$. Thirone et al também relataram um caso semelhante, onde a paciente apresentava oligossintomática e teve sucesso após instituído o tratamento com a LT4 ${ }^{14}$. Já Neves et al, relatou um caso, onde a paciente apresentou significativamente sintomática, sendo necessária a pericardiocentese ${ }^{15}$.

A paciente apresentava em bom estado geral e oligossintomática e omitiu, durante o início da internação, seu histórico de hipotiroidismo, só revelando 5 dias após. Seu ecocardiograma inicial não evidenciou prejuízo na função cardíaca, provavelmente explicando a pobreza de sintomas da paciente, mas mostrou um volumoso derrame pericárdico. Não houve necessidade de pericardiocentese, pois a mesma encontrava-se estável, do ponto de vista hemodinâmico. Este exame é um instrumento importante para o diagnóstico e mensuração do volume e repercussão hemodinâmica da efusão pericárdica. Instituído o tratamento com a levotiroxina sódica, houve melhora da astenia. Ao longo do tempo, com a otimização da terapêutica, ocorreu a normalização do TSH, assim como a dos exames de imagem. No entanto, a remissão do derrame é insidiosa, de modo que poderá estar presente por vários meses, ou até anos, mesmo após a instituição da terapêutica ${ }^{16,17}$.

Portanto, é importante saber que medidas agressivas para o derrame, podem ser ineficazes em alguns casos, e que a melhor conduta, neste caso, foi a reposição com levotiroxina para restabelecer o eutiroidismo.

Casos de doentes com psoríase e tireoidite de Hashimoto (TH) são descritos ${ }^{18,19}$, sendo esta (TH) uma doença mediada por células Th, tal como a psoríase ${ }^{19}$. Estudos recentes apontam para um papel importante dos hormônios tireoidianos na hiperproliferação epidérmica nas placas de psoríase ${ }^{20}$.

Também é frequente o achado de auto-anticorpos (FAN e anti-ss-DNA) em pacientes com TH e doença de Graves, mas sem qualquer evidência clínica no lúpus eritematoso sistêmico (LES) ou positividade para auto-anticorpos específicos, como anti-Sm e anti-DNA nativo $^{21,22}$. Observa-se no caso exposto que a paciente 
apresentou a positividade para o FAN, mesmo em nível baixo, junto da psoríase e tireoidite de Hashimoto.

\section{CONCLUSÃO}

O presente trabalho mostra como pode ser diversa a apresentação de doenças sistêmicas, em especial do hipotireoidismo, cuja fisiopatologia pode fornecer ao médico assistente desde quadros mais comuns como manifestações mais raras. Com isso, os autores deste relato buscam ressaltar a importância de uma boa propedêutica afim de que se chegue à melhor elucidação e terapêutica na prática clínica. Apesar de desafiador, principalmente diante dos poucos relatos desse tipo de apresentação, este trabalho mostra como um paciente, mesmo com alterações significativas de marcadores tireoidianos pode permanecer oligossintomático ou mesmo assintomático.

Participação dos autores no texto: Tiago Cardoso Martinelli - Elaboração do texto escrito, introdução e relato de caso, submissão do artigo, revisão pré submissão. Renan Corrêa Braga - Responsável pela elaboração do texto escrito, introdução e relato de caso. Bárbara Ahnert Blanco de Moura Magalhães - Responsável pela elaboração do texto escrito, discussão e conclusão, e auxílio na submissão do artigo. Sofia Villela Musso - Auxílio na elaboracão do texto escrito, discussão, e ajuste do texto para língua inglesa. Regina Célia Tonini - Auxilio no estudo do caso clínico e descrição no relato de caso. Rachel Torres Sasso - Auxilio no desenvolvimento teórico na discussão do trabalho. Carmen Dolores Gonçalves Brandão - Endocrinologista, Orientadora do trabalho.

\section{REFERÊNCIAS}

1. Freitas MC, Lima LHC. Diagnóstico e tratamento do Hipotireoidismo. In Vilar, Lúcio. Endocrinologia clínica. 5a ed. Rio de Janeiro: Guanabara Koogan; 2013. p.288-9.

2. Chaker L, Bianco AC, Jonklaas J, Peeters RP. Hypothyroidism. Lancet. 2017;390:1550-62. doi: 10.1016/ S0140-6736(17)30703-1.

3. Caturegli P, Remigis AD, Rose N. Hashimoto thyroiditis: Clinical and diagnostic criteria. Autoimmun Rev. 2014;13(45):391-7. doi: 10.1016/j.autrev.2014.01.007.

4. Almandoz JP, Gharib H. Hypothyroidism: etiology, diagnosis, and management. Med Clin North Am. 2012;96(2):203-21. doi: 10.1016/j.mcna.2012.01.005.

5. Kostoglou-Athanassiou I, Ntalles K. Hypothyroidism - new aspects of an old disease. Hippokratia. 2010;14(2):82-7. Disponível em: https://www.ncbi.nlm.nih.gov/pmc/articles/ PMC2895281/

6. Udovcic M, Pena RH, Patham B, Tabatabai L, Kansara A. Hypothyroidism and the Heart. Methodist DeBakey Cardiovasc J. 2017;13(2):55-9. doi: 10.14797/mdcj-13-255.

7. Jabbar A, Pingitore A, Pearce SHS, Zaman A, Iervasi G, Razvi S. Thyroid hormones and cardiovascular disease. Nature Rev Cardiol. 2016;14(1):39-55. doi: 10.1038/ nrcardio.2016.174

8. Patil VC, Patil HV, Patil VA, Sanjay P. Cardiac tamponade in a patient with primary hypothyroidism. Indian J Endocrinol Metab. 2011;15(Suppl 2):S144-6. doi: 10.4103/22308210.83358 .

9. Mokta J, Mokta K, Panda P, Sharma M, Bhatia V. A swinging heart. Indian J Endocrinol Metab. 2013;17(Suppl 3):S660-1 doi: $10.4103 / 2230-8210.123560$.

10. Olearczyk BM, Chongkrairatanakul T, Menzies DJ. The swinging heart: Cardiac alternans and right ventricular collapse in classic tamponade. Can J Cardiol. 2009;25(4):240. doi: 10.1016/S0828-282X(09)70080-7.

11. Garber J, Cobin R, Gharib H, Hennessey J, Klein I, Mechanick J, et al. Clinical Practice Guidelines for Hypothyroidism in Adults: cosponsored by the American Association of Clinical Endocrinologists and the American Thyroid Association. Endocrine Pract. 2012;18(6):9881028. doi: 10.4158/EP12280.GL.

12. Smotar EM, Rubin JE, Avramides A, Carter AC. Cardiac tamponade in primary myxedema and review of the literature. Am J Med Sci. 1976;272(3):345-52.

13. Karu A, Khalife W, Houser R, VanderWoude J.
Impending cardiac tamponade as a primary presentation of hypothyroidism: case report and review of literature. Endocrine Pract. 2005;11(4):265-71. doi: 10.4158/ EP.11.4.265.

14. ThironeACP,DanieliRV, RibeiroVMFC.Derramepericárdico maciço como manifestação inicial do hipotireoidismo. Arq Bras Endocrinol Metab. 2012;56(6):383-7. doi: 10.1590/ S0004-27302012000600007.

15. Neves AIA, et al. Derrame pericárdico como forma de apresentação do hipotireoidismo secundário à radioablação com iodo. Rev Bras Cardiol. 2011;24(4):25861. Disponível em: http://sociedades.cardiol.br/socerj/ revista/2011_04/a_2011_v24_n04_07relato01.pdf.

16. Lin C, Liu C, Lin T, Chen C, Chen B, Lin C. Myxedema associated with cardiac tamponade. Jpn Heart J. 2003;44(3):447-50. doi: 10.1536/jhj.44.447.

17. Omura $Y$, Ugi S, Sugimoto $T$, Nishio $Y$, Maegawa $H$, Kashiiwagi A. Massive pericardial, effusion secondary to Hashimoto's disease. Eur J Intern Med. 2007;18(5):438-40. doi: 10.1016/j.ejim.2007.05.001.

18. Saeed M, Brown GE, Agarwal A, Pellowski D, Jacks J, Liverett HK, Vyas KS. Autoimmune clustering: Sweet syndrome, Hashimoto thyroiditis, and psoriasis. J Clin Rheumatol. 2011;17(2):76-8. doi: 10.1097/ RHU.0b013e31820e624f.

19. Giménez-García R1, Sánchez-Ramón S, Cuellar-Olmedo LA. Palmoplantar pustulosis: a clinicoepidemiological study. The relationship between tobacco use and thyroid function. J Eur Acad Dermatol Venereol. 2003;17(3):276-9. doi: 10.1046/j.1468-3083.2003.00510.x.

20. Borges AS, Brasileiro A. Disfunção tiroideia: uma nova associação com a psoríase? Rev SPDV. 2018;75(3):259-63. doi: $10.29021 /$ spdv.75.3.814.

21. Katakura M, Yamada T, Aizawa T, Hiramatsu K, Yukimura Y, Ishihara M, Takasu N, Maruyama K, Kameko M, Kanai $\mathrm{M}$, et al. Presence of antideoxyribonucleic acid antibody in patients with hyperthyroidism of Graves' disease. J Clin Endocrinol Metab. 1987;64(3):405-8. doi: 10.1210/jcem64-3-405.

22. Baethge BA, Levine SN, Wolf RE. Antibodies to nuclear antigens in Graves' disease. J Clin Endocrinol Metab. 1988;66(3):485-8. doi: 10.1210/jcem-66-3-485.

Recebido: 01.09.18

Aceito: 22.03 .19 